E.L.U.A. 2, 1984, págs. $61-89$

\title{
MULTILINGÜISMO Y MULTILECTISMO *
}

\author{
FRANCISCO GIMENO MENÉNDEZ \\ (Universidad de Alicante)
}

\section{Introducción}

Los fenómenos de multilingüismo -o bilingüismo, en generaレson demasiado complejos y diversos para ser interpretados desde una perspectiva disciplinaria. Inicialmente podríamos asumir que la repartición de los usos lingüisticos es social y su descripción corresponde a la sociología, la filiación diferencial de sus aplicaciones es lingüística, y el análisis de sus polos de repartición corresponde a la psicología de la comunidad de habla y del hablante. Asimismo otros aspectos del problema, como las cuestiones pedagógicas, politicas, juridicas, geográficas y culturales no deberian faltar en cualquier reflexión seria.

Nuestra aproximación se inscribirá dentro de un marco más explicativo que descriptivo, a fin de disponer de una visión sociolingüística óptima del problema. Nuestro propósito será un análisis y revisión critica del multilingüismo y multilectismo a la luz de una sociolingüística general, y corroborar la viabilidad de una estricta teoría sociolingüística del multilingüismo y multilectismo, bajo unas coordenadas formales $y$ explicativas (v. Gimeno, 1979, págs. 138 ss.).

* Una primera versión de este trabajo fue presentada en el XVI Congreso Internacional de Lingüistica y Filologia Románicas, Palma de Mallorca, 7-12 de abril de 1980. 
1.1. En principio, el análisis sociolingüístico del comportamiento bilingüe es dependiente tanto de los modelos pragmáticos de actuación comunicativa, como de una teoria interdisciplinaria. El individuo debe ser considerado como miembro de la comunidad de habla, que se caracteriza por un conocimiento compartido de las restricciones comunicativas y de las normas del comportamiento social. El concepto de competencia sociolingüistica parece ser el más prometedor para el establecimiento de un modelo explicativo de la comunicación, dentro de la comunidad de habla bilingüe. Es preciso subrayar, por otra parte, que multilingüismo es un concepto integrador, relativo y neutro que no determina el grado de competencia ni la frecuencia de uso variable, como ni siquiera el ámbito de las distintas lenguas particulares, ni la diferenciación social y/o funcional entre ellas.

Mas también debemos de entender el contacto de lenguas en su acepción más amplia. En este sentido no hay, en rigor, diferencia esencial entre dos variedades de una misma lengua histórica y dos lenguas históricas diferentes. La diferencia es sólo de grado de diversidad: dentro de una lengua particular, las diferencias son menores que entre esta misma lengua y otra lengua particular, $y$, normalmente, no afectan a todo el componente fonológico, proceso transformativo o primera entrada del lexicón, sino a secciones más o menos amplias de estos procesos, según los casos. $Y$ aun cuando en el pasado se ha extendido técnicamente el término de bilingüismo o multilingüismo para cubrir también esos casos de contacto, caracterizamos propiamente dicha situación como multilectismo, que comprendería tanto los diversos lectos o variedades de la misma lengua particular, como varias lenguas particulares distintas, y diversos lectos de diferentes lenguas particulares. Una gramática multilectal, basada en los presupuestos sociolingüísticos del multilingüismo y multilectismo, permite la teoría más adecuada a la lingüística histórica (v. Gimeno, 1983a; 1983c; 1984a; 1985).

1.2. Las alusiones a influencias entre las lenguas no fueron ajenas a los lingüistas del siglo pasado. Así, por ejemplo, $\mathrm{H}$. Paul las consideraba como hechos externos a las lenguas y, en cualquier caso, como fenómenos marginales. Sin embargo, H. Schuchardt (1885) planteó la diferenciación del contacto multilectal como fuente de excepciones a las leyes fonéticas, propiciando las indagaciones dialectológicas y sociolingüisticas. Frente al desarrollo de las primeras, hemos asistido al considerable retraso de las segundas, hasta los años cincuenta de nuestro siglo. Varias razones pueden darse sobre la marginación y resurgimiento de la investigación bilingüe: 
a) las propias exigencias metodológicas del estructuralismo lingüistico, las cuales preservaron la integridad y funcionamiento del mecanismo interno de la lengua de cualquier referencia a variables externas;

b) la profunda transformación del mapa político internacional, después de la segunda guerra mundial, la cual ha provocado el multilingüismo de muchos países con todas las secuelas sociales, culturales y escolares, y la consiguiente política lingüistica, y

c) la oposición de las minorias étnicas y nacionalistas a la integración en los programas de aculturación y asimilación.

Los estudios sociolingüisticos del multilingüismo se han centrado sobre los aspectos lingüísticos y sociales del problema, influenciados fuertemente por las contribuciones teóricas de U. Weinreich (1953) y Ch. A. Ferguson (1959). La alternancia entre variedades gramaticalmente distintas ha originado investigaciones encaminadas a conocer dónde y bajo qué condiciones existe interferencia o "cambio de norma", bien a través de la cantidad de formas híbridas en muestras de habla obtenidas, bien a partir de los informes facilitados por los propios bilingües sobre las actitudes hacia las variedades lingüísticas. Dichas alternancias estarían condicionadas ampliamente por una compleja interdependencia de los factores presentes en la organización social de la comunidad y en el contexto social de la comunicación.

Últimamente se han establecido dos fases del multilingüismo basadas en la circunscripción a un comportamiento individual o a fenómeno social. En particular, J. A. Fishman (1969) distingue ambos aspectos como tesis y antitesis, y propone una tercera prospectiva final, síntesis de la microsociología y macrosociología, la cual caracterizaría el bilingüismo de la década de los setenta. Por mi parte, en los años ochenta, seguiré una casuística comprensiva, desde un punto de vista explicativo ( $v$. Gimeno, 1981), y destacaré tres direcciones:

a) lenguas en contacto, cuya aproximación se basa en medidas de interferencia y análisis contrastivos de las lenguas en tensión, a partir de modelos analíticos derivados del estudio de comunidades monolingües, y asumiendo que la estructura de las lenguas implicadas es relativamente uniforme y conocida;

b) diglosia, asentada sobre la integración del bilingüismo individual dentro de las normas sociolingüisticas de la comunidad de habla, y el reconocimiento de la diferenciación social y funcional de las lenguas o variedades para la comunicación interior del grupo, y

c) regla variable o variacionista, cimentada en la sociolingüística 
última, la cual sugiere la posibilidad de explicar la competencia sociolingüística de las comunidades de habla bilingües en los repertorios lingüísticos, integrando un conjunto ordenado de reglas variables, bajo la forma de probabilidades teóricas.

\section{Lenguas en contacto}

El concepto de bilingüismo se ha dilatado desde principios de siglo, y se ha considerado, durante mucho tiempo, como el igual dominio de dos lenguas. Varias definiciones pueden destacarse como jalones de las vicisitudes de nuestro concepto, desde Bloomfield (1933, pág. 64): "control de dos lenguas como si ambas fueran maternas", hasta Diebold (1961, pág. 111), que sugiere la ampliación del concepto para incluir el simple conocimiento pasivo de la segunda lengua. Para Weinreich (1953, pág. 7), bilingüismo o multilingüismo es sinónimo de lenguas en contacto y se caracteriza por la práctica de utilizar alternativamente dos o más lenguas por las mismas personas. Actualmente, Mackey (1976, pág. 10) considera que el estudio del bilingüismo desborda el interés lingüístico y aparece sujeto a la distinción entre bilingüismo, como fenómeno individual, y contacto interlingüistico, como manifestación de grupo.

Sin embargo, la propuesta metodológica de oponer la observación «microscópica» de los fenómenos de contacto en el comportamiento de los individuos bilingües, al estudio «macroscópico» de acción de una lengua sobre otra fue ya sugerida por Weinreich (1968, pág. 654). Es más, en extremo, cualquier dicotomía entre individuo y grupo seria perjudicial, ya que el bilingüe, último recinto del contacto de lenguas, se encuentra condicionado por los factores sociocuiturales de la comunidad, así p. ej. el orden, la edad de aprendizaje, la habilidad relativa de las lenguas, etc. son predeterminados frecuentemente a los hablantes por su propia comunidad.

2.1. Haugen (1973, pág. 521) define al bilingüe ideal como aquél que domina las reglas de la gramática y el léxico de dos distintas comunidades lingüísticas, $y$, por lo tanto, posee una doble competencia lingüística. No obstante, ciertas coincidencias en la sustancia de la expresión y del contenido sugieren una identificación hibrida al bilingüe, llamada matriz de confusión, y de ahí los paralelismos particularmente extensivos entre las lenguas que han estado en intenso contacto. El resultado será la convergencia de códigos como clave de la 
actuación bilingüe, o con otras palabras, la amalgama gradual de dos códigos hacia un diasistema integrador. Asistimos, pues, a una clara percepción de la distinción entre bilingüismo ideal y bilingüismo real, y la contradicción inherente de una misma metodologia.

Se habia distinguido ya langue y parole, sobre todo en el campo de la interferencia, pero es Mackey (1962), quien plantea la dicotomía saussureana más especificamente. El bilingüismo, reducido propiamente a una manifestación individual, no será un asunto del código sino una característica de su uso, es decir, si la lengua es propiedad del grupo, el bilingüismo debe materializarse a través de las interacciones individuales. En definitiva, la descripción del bilingüismo comprenderá un modelo complejo de las actuaciones bilingües, lingüísticamente interrelacionadas, las cuales varían en grado, función, alternancia e interferencia (v. Mackey, 1976; Granda ', 1978; Alpera, 1981; Quilis et al., 1982; Azurmendi ${ }^{2}$, 1983; Gimeno, 1975).

En el análisis de los contactos interlingüísticos, la hipótesis de una sincronía, la cual ha sido extremadamente útil para establecer gramáticas abstractas descriptivas o explicativas, muestra limitaciones sobre todo cuando se trata de describir sistemas inestables y pasajeros de

' Postula, entre otras, una relación indirecta entre las lenguas africanas y el español de América, desde y a través de un estadio intermedio criollo, por medio de un proceso más o menos Jento de reestructuración y relexificación hacia la lengua dominante, el cual implicaria un continuum postcriollo y una descriollización lingü istica con las variedades sucesivas de basilecto criollo, mesolecto y acrolecto castellano. Con respecto al caso de la superposición de una lengua prestigiosa heterogénea o alejada respecto a un habla criolla, opina que nunca podría gestarse un continuum postcriollo, sino la paulatina relegación y desuso acelerado del criollo, entre coordenadas de normalización y diglosia (págs. 399 ss.). Posteriormente, Granda (1980) ha confirmado la sugerencia de U. Weinreich (1953) sobre la posibilidad de préstamos morfológicos entre lenguas, frente a la tesis sotenida por A. Meillet y $L$. Tesniere. A pesar de la muy diferente tipologia de la lengua guarani y castellana (aglutinante la una, flexiva la otra), la extensión y relevancia de los préstamos morfológicos del guarani en el castellano paraguayo denotan un continuum que explica las diferentes modalidades de cambio de norma (code-switching) y una clara -aunque poco probabletendencia hacia la constitución de una lengua mixta.

2 Ha planteado la elaboración de un modelo idóneo para la descripción sociolingüística del bilingüismo éuskaro-castellano y su aplicación parcial a la población escolar de $5 .^{\circ}$ y $8 .^{\circ}$ curso de E.G.B., escolarizada en las ikastolas, en la comarca de San Sebastián. Entre las variables lingüisticas seleccionó: a) productividad lingüistica cuantitativa, b) grado de lexicalización y de la clase léxica, c) distancia interlingüistica, d) corrección lingüistica y e) divisiones significativas o semánticas. Entre las variables sociales tuvo en cuenta: a) factor geográfico, b) edad, c) sexo, d) clase de escolarización, e) variedad primaria, f) variedad del ámbito familiar, g) variedad más frecuente con los amigos y h) variedad de encuesta. En el tratamiento estadístico aplicó el método de análisis univariante y multivariante, a través de diferentes técnicas estadisticas y, en particular, del análisis en componentes principales. 
comunidades heterogéneas y de lenguas en contacto. En líneas generales, la coexistencia de sistemas debe inscribirse dentro de las soluciones siguientes, aunque no se excluye una cierta relación entre ellas en determinadas situaciones:

a) sustitución lingüistica, o abandono de una lengua particular (A) en beneficio de otra (B);

b) cambio de norma, o uso alternativo de dos lenguas en el mismo enunciado, oración o constituyente, y

c) amalgama, o reestructuración lingüistica de dos lenguas particulares en una nueva gramática.

2.2. Actualmente hay en Europa, según Vildomec (1963, págs. 230 ss.), dos tipos principales de multilingüismo: 1) el propio de las masas populares en algunas regiones y ciudades, especialmente común en ciertas partes de la Europa del Este, en la que ha tenido lugar mucha migración y algunos cambios de fronteras, y 2) el multilingüismo de élites educadas y conectadas principalmente a la facultad y educación universitaria, particularmente frecuente en los pequeños países centrales. Las principales consecuencias lingüisticas del bilingüismo, según su gran experiencia en este tipo de estudios, son:

a) influencias reciprocas en la actuación lingüistica del multilingüismo, tanto en la lengua materna como en las otras lenguas;

b) los conceptos de lengua materna y lengua estándar o adquirida son de capital importancia;

c) la similitud mutua de dos o más lenguas juega una parte importante, no sólo en el habla de un multilingüe actual, sino también en la historia de las lenguas;

d) las influencias extranjeras en los subsistemas fonológicos, morfológicos y sintácticos de una lengua son una prueba más segura de la existencia de multilingüismo en el pasado, que los meros préstamos de vocabulario, y

e) una comunidad de habla multilingüe es un vivero de sociolectos multilingües.

En una visión global de las publicaciones multilingües de la Europa occidental, entre los años 1918 y 1968, cabe destacar la gran cantidad e importancia de las investigaciones sobre sustrato, superestrato y adstrato, y la ausencia considerable de estudios sobre el fenómeno multilingüe en sujetos individuales. Aun así, y dentro de este último, el 
campo más activo del bilingüismo europeo ha sido el apartado de la interferencia lingüistica y préstamo, seguido del grupo de descripciones contrastivas y amalgamas de lenguas. Es más, la mayoría de los análisis de interferencia léxica son trabajos ricos en observaciones empíricas, pero pobres en sistematizaciones teóricas, y se caracterizan por una perspectiva atomista de las lenguas, especialmente antes de 1950 (Oksaar, 1972, págs. 486 ss.).

2.3. La crítica al modelo de «lenguas en contacto" se ha centrado sobre su miopía y marginalidad del fenómeno bilingüe, puesto que presentó la categoría de monolingüe como única base natural y estable de interacción social, y caracterizó al bilingüismo como reflejo de la comunicación entre grupos monolingües. Deficiencias semejantes encontrariamos entre la investigación psicológica y sociológica tradicional del bilingüismo. Para Fishman (1969, pág. 275) ha sido, en parte, consecuencia de generalizar prematuramente la limitada experiencia occidental, que confundia el bilingüismo con algunos de sus atipicos concomitantes: inmigración a gran escala, rupturas sociales o individuales, etc. La interferencia no es un baremo absoluto y nocivo del multilingüísmo, sino variantes y variables relativas a ciertos subgrupos y circunstancias, regidas por las normas sociolingüisticas de la comunidad de habla, las cuales deben ser descubiertas por el investigador.

Cabria preguntarse qué otro punto de partida - no de llegadadeberia haberse planteado si no el rendimiento lingüistico de los grupos monolingües. Porque el modelo tradicional de lenguas en contacto ha sido una primera fase, donde se han mostrado claramente, como ha puesto de manifiesto Mackey (1969, pág. 365), las propias deficiencias de las técnicas sincrónicas convencionales de descripción y la necesidad de nuevas teorías y métodos, en particular la contribución de una cooperación interdisciplinaria, técnicas cuantitativas, competencia comunicativa, etc. Frente a un estrecho bilingüismo motivado por estrategias verbales, predice un multilingüismo gobernado por reglas de una gramática, que incluyera las lenguas en contacto con conjuntos de normas circunscritas a situaciones sociales particulares. Los trabajos últimos de esta linea metodológica ( $v$. supra) van superando ampliamente aquellas deficiencias.

\section{Diglosia}

Supone una ruptura con los modelos tradicionales de «lenguas en 
contacto». El estudio del bilingüismo, en esta dirección, se plantea la descripción sociolingüistica dentro de una comunidad de habla bilingüe, a través de las normas sociales que determinan el uso de variedades particulares, entre singulares individuos y en situaciones especificas. De ahí que la auténtica dimensión del individuo bilingüe sea manifestar las normas sociolingüisticas de su particular subgrupo. Estas investigaciones microsociolingüísticas deben complementarse con el desarrollo de técnicas cuantitativas que nos permitan medir y describir el bilingüismo extenso y relativamente estable, de una forma interdisciplinaria y clarificadora.

3.1. Una posible solución es presentada por el modelo sociolingüístico integrado de Fishman (1968a), quien aboga por una teoria descriptiva-predictiva que partiria de parámetros de un ordenamiento superior (comunidad de habla y ámbitos del comportamiento lingüístico) a parámetros de un ordenamiento inferior (dimensiones de las relaciones sociales y tipos de interacción). El cambio en la esfera de estas categorías muestra cómo cambia la variedad lingüistica, en el contexto de qué dimensiones sociales y en qué etapas temporales.

En el orden superior de análisis, particularmente en el nivel más elevado de la abstracción, el término “diglosia», del francés diglossie, parece ser más apropiado que bilingüismo. Bilingüismo se reservaria para caracterizar una situación propia de la conducta individual, mientras que diglosia haria referencia a un tipo de organización sociolingüistica basada primariamente en la asignación social de las funciones a distintas lenguas o variedades. Mientras el primero se orientaria hacia propósitos intercomunitarios, la segunda enlazaría con el bilingüismo social dentro de la comunidad de habla.

3.1.1. La definición clásica de diglosia fue dada por Ferguson (1959, pág. 336) como «una situación lingüística relativamente estable en la que, al lado de los nativos geolectos de la lengua (que pueden incluir una variedad estándar o varios estándares regionales), existe una variedad superpuesta muy divergente, altamente codificada (con frecuencia gramaticalmente más compleja), vehículo de un amplio y respetado cuerpo de literatura escrita (ya de un periodo antiguo, ya de otra comunidad lingüística), la cual se aprende ampliamente en la educación formal y se utiliza para la mayor parte de los usos escritos y en el hablar formal, pero que no se emplea por ningún sector de la comunidad en la conversación ordinaria». 
Este enfoque inicial fue generalizado por Fishman (1968b, págs. 135 ss.) a las comunidades bilingües, y comprende así bajo diglosia cualquier situación en la que se da un sociolecto alto (A), vehículo normal en la comunicación más culta o formal, y un sociolecto bajo (B), empleado en la interacción no formal o familiar. Sin embargo las situaciones de bilingüismo y diglosia pueden ser analizadas conjuntamente y constituirian formas de distribución en los modelos del comportamiento sociolingüístico.

El bilingüismo es independiente del valor funcional de las lenguas implicadas en el repertorio lingüístico. La diglosia, en cambio, implica la existencia de sociolectos funcionalmente diferenciados y, en principio, es indiferente que esos sociolectos pertenezcan a la misma o a distintas lenguas. Bilingüismo y diglosia pueden, por lo tanto, coincidir cuando los sociolectos y registros sean lenguas diferentes. De acuerdo con ello, podrian distinguirse cuatro posibles configuraciones, cuya adecuada explicación sólo se alcanzará con análisis de los desarrollos históricos de las comunidades bilingües: 1) diglosia y bilingüismo, 2) bilingüismo sin diglosia, 3) diglosia sin bilingüismo, y 4) ni diglosia ni bilingüismo.

3.2. El fenómeno del bilingüismo diglósico se ha materializado con el estudio de Fishman, Cooper, Ma y otros sobre el barrio puertorriqueño de la ciudad de Jersey, New Jersey (1971). En él se conformaron ocho técnicas de medidas alternativas de bilingüismo y se realizaron dos análisis estadísticos de varianza, análisis de factores y de regresión múltiple (v. págs. 483-512), en relación con cuatro variables lingüísticas globales (interferencia, lectura bilingüe, y alcance del repertorio en inglés y en español) y seis variables demográficas (edad, sexo, lugar de nacimiento, instrucción, profesión y años de residencia en los Estados Unidos continentales). Dichas medidas resultaron de la intersección de tres parejas (v. fig. 1), que agrupaban las distintas facetas disciplinarias: a) pruebas de sondeo (clasificación a priori y deducción empírica), b) tipos de comportamiento (habilidad y uso), y c) fuentes de observación (propia actuación del informante e informe retrospectivo). Se trata, en suma, de una técnica general para proporcionar al investigador del bilingüismo unos componentes mínimos de trabajo en cualquier estudio particular.

El hecho de que los ámbitos puedan aproximarse a un cierto comportamiento lingüístico de los informantes, nos sitúa ante un posible nivel sociolingüístico, útil y eficaz, para la descripción de los modelos sociales de habilidad y uso bilingüe. Ellos no son tan neutros como la 


\begin{tabular}{|c|c|c|c|c|c|}
\hline \multirow{2}{*}{} & \multicolumn{4}{c|}{ Pruebas de sondeo } \\
\cline { 2 - 6 } & \multicolumn{2}{|c|}{ A priori } & \multicolumn{2}{c|}{ Empírica } \\
\cline { 2 - 6 } & Habilidad & Uso & Habilidad & Uso \\
\hline \multirow{2}{*}{$\begin{array}{l}\text { Fuente de } \\
\text { observación }\end{array}$} & Actuación & 1 & 2 & 5 & 6 \\
\cline { 2 - 6 } & Informe & 3 & 4 & 7 & 8 \\
\hline
\end{tabular}

Figura 1.-Clasificación de técnicas para la medida del bilingüismo diglósico.

estratificación social de la comunidad, ni tan efímeros como la situación. La futura investigación sociolingüística deberá demostrar la validez de la deducción de ámbitos, al relacionar la estructura sociocultural con situaciones sociales manifiestamente coherentes.

Finalmente, las aportaciones lingüísticas se limitaron, ante las dificultades de documentar los modelos de interacción entre la elección de lengua y el contexto social, a sugerir la hipótesis de la relación entre los ámbitos de uso de una variedad lingüística y la mayor diversidad de la variación funcional o estilistica. Son significativas, además, las implicaciones de la variación, ya funcional, ya demográfica, en el planteamiento cuantitativo independiente de las variables españolas e inglesas dentro de la comunidad de habla puertorriqueña (cfr. págs. 465 ss.). La coocurrencia de variables demográficas y funcionales no debe ser considerada, pues, un rasgo necesario de una comunidad de habla, sino un grado de interacción y de complejidad de la estratificación.

3.3. El Estado español es uno de los más importantes conjuntos multilingües y de base cultural más heterogénea de Europa. Catalán y castellano son, como lenguas románicas, ramas de un mismo tronco, aunque totalmente independientes, dentro de una cierta proximidad geográfico-histórico-cultural. Es frecuente encontrar en obras de autores castellanos la afirmación de la doble competencia lingüistica de los catalanes, ya que poseen dos lenguas propias, el catalán y el castellano. Desde otro punto de vista, els Paisos Catalans se han considerado bilingües dada la mixtura de poblaciones de habla catalana y castellana. Sin embargo, como ha puesto de manifiesto Aracil (1966, pág. 19), se tiene la conciencia de cierta carga negativa ideológica en el 
término bilingüismo, puesto que refleja, por una parte, una situación real de conflicto, y por otra, neutraliza sus inevitables tensiones.

Entre las diversas clases de bilingüismo en la sociedad catalana, Badia (1964, pág. 149) distingue entre bilingüismo natural y ambiental. El primero caracterizaría a los hijos de padres de diferentes lenguas, o aquéllos que se encuentran en contacto con dos lenguas desde la cuna, mientras que el segundo mostraria la convivencia de dos lenguas, una oficial y otra minoritaria, como consecuencia de diversos hechos históricos desde hace varios siglos, en particular, el decreto de Nueva Planta (1716), bajo el reinado de Felipe V. Desde entonces, el divorcio de lengua y cultura ha sido general, con una serie de vaivenes en ese proceso, aunque bajo unas circunstancias más penosas desde 1939 (v. Badia, 1969; 1972; 1977).

Bilingüismo, efectivamente, no es la respuesta adecuada al uso de dos lenguas en la comunidad catalana, si el alcance de los conceptos viene determinado por la situación contextual. Y si partimos del conflicto diglósico catalán como relación de elementos que pueden caracterizarse por una oposición de tipo objetivo o subjetivo, bien consciente o bien impuesta por las circunstancias, solamente podrá ser enunciado afrontando las coordenadas sociales, económicas, políticas y culturales (v. Badia, 1974; Kremnitz, 1979; Gimeno, 1982a; 1982b).

3.3.1. Conviene precisar, sin embargo, un punto decisivo en la reelaboración y superación del concepto de diglosia. La noción de conflicto lingüistico activa la configuración estática y estable de la diglosia, y se constituye en un proceso histórico complejo y de larga duración, en el que lenguas y hablantes se encuentran inmersos. Aracil (1965; 1979a; 1979b) entiende el conflicto lingüistico como aquella coyuntura en la que surge el dilema entre el proceso de normalización y el de sustitución lingüistica. La normalización lingüística consiste sobre todo en elaborar y poner en vigor sistemas de normas de uso lingüístico, y no puede limitarse nunca a los aspectos puramente lingüísticos, sino que ha de tener en cuenta una gran cantidad de factores sociales y políticos. La sustitución lingüistica es la redistribución de las variedades que forman el repertorio lingüístico de un ámbito sociocultural. El desplazamiento implica contacto de lenguas, y es cuantitativo (en términos de número de hablantes y frecuencias de uso) y/o cualitativo (en términos de ámbitos diferenciales y normas de uso). De hecho los mecanismos del proceso revelan cómo las frecuencias y las normas se generan reciprocamente.

Por su parte, Ninyoles $(1972 ; 1976 a ; 1977)$ ha replanteado una lla- 
mada «diglosia estructural», que establece la superposición de dos idiomas y su relación e interdependencia con el resto de los rasgos estructurales (sociales, económicos, culturales y políticos) de una sociedad. Dicha diglosia presupone una dualidad ideológica valorativa y un desequilibrio real, provocando un conflicto lingü ístico que se mueve históricamente entre coordenadas de normalización y sustitución. Esta última, y en el caso de lenguas próximas, ofrecería un proceso de paulatina dialectalización del sociolecto bajo (B), mediante su fragmentación indefinida en innumerables lectos locales y confinamiento a mero registro familiar o casual (v. Kloss, 1967; Lafont, 1977).

En suma, la pauta diglósica ha tenido una considerable aceptación en la sociolingüística catalana, y puede decirse que actualmente es una de las tendencias más desarrolladas y reelaboradas. Vallverdú (1970; $1980 ; 1981$ ) ha examinado la diversa concepción de los sociolingüistas catalanes, especialmente la dispersión interpretativa sobre la escueta e insuficiente teoría de Fishman, y se sitúa dentro de una consideración de las diferencias sociales y culturales, para analizar el conflicto lingüistico catalán en las diferentes comunidades de habla.

3.4. La crítica al modelo de "diglosia» se ha efectuado sobre las diferencias del concepto de diglosia en Fishman y Ferguson. Las diferentes lenguas implicadas, según Dittmar (1973, págs. 176 ss.), comportarian algo más que distinciones de grupos sociales, puesto que factores externos de coherencia nacional e histórica desempeñarian un importante papel en las actitudes adoptadas por tales comunidades lingüisticas, sobre las propias de unas variedades de una misma lengua. La clasificación de bilingüismo y diglosia, aunque es un criterio central para la clasificación de las comunidades multilingües, aparecería superficial, manifiestamente ambigua y hasta ocasionalmente contradictoria.

Por mi parte, plantearía que los estudios sociolingüísticos deben apoyarse en una coherencia y homogeneidad metodológica, que no siempre encontramos en los estudios diglósicos (v. Gimeno, 1984d). Al mismo tiempo, la comunidad de habla bilingüe debe caracterizarse por una diferenciación social y/o funcional de las distintas lenguas o variedades. Es decir, aparte de la diversificación funcional de los sociolectos, sería útil tener en cuenta la posible correlación de los diversos subgrupos sociales con la distribución de las variables lingüísticas, circunstancia eludida, a priori, en el bilingüísmo de los puertorriqueños, puesto que la descripción sociolingüística se limitó a un sector del grupo socioeconómico bajo (cfr. Fishman, Cooper, Ma etal., 1971, págs. 517 s.). 
Por otro lado, la prevención sobre las propias implicaciones lingü isticas de competencia y actuación sociolingüisticas, en favor de abstracciones sociológicas más rudas e inseguras, simplifican el problema central de la comunidad de habla. En particular, Fishman (1968a, págs. 566 ss.) ha basado la identificación y ejecución de los valores culturales en la situación de diglosia, sobre el establecimiento de unos parámetros inductivos de valores de comportamiento y de actitud. Del mismo modo, resulta un nivel sociológicamente perspicaz, pero todavía superficial y excesivamente elemental, la inducción de ámbitos identificados culturalmente. Con todo, las distinciones de Fishman aparecen como interesantes tipologías del multilingüismo extenso y relativamente estable, en entornos sociales amplios y complejos.

\section{Regla variable o estudio variacionista}

Se entiende por bilingüismo o multilingüismo, dentro de este enfoque, la covariación de dos o más lenguas en el repertorio lingüistico de una comunidad de habla. El problema se plantea en determinar si la actuación del bilingüe debe analizarse antes como una comunicación funcionalmente estructurada dentro de una comunidad de habla que como una mera interacción entre códigos monolingües. Con otras palabras, si las variedades sociales y/o funcionales del bilingüe forman parte de sistemas lingüísticos separados, o de un diasistema complejo con variación interna. La existencia de convergencia no puede ya ponerse seriamente en duda, asi como la distancia entre lenguas no es un valor constante, sino una variable en función de la intensidad del contacto, (sub)grupo y contexto social. En todo caso, cabe referirse más a diferencias de creencias y actitudes sociales hacia las variedades lingüisticas que a divergencias entre estructuras lingüisticas, si todas las lenguas particulares coinciden probablemente en el nivel más abstracto de la estructura profunda.

4.1. Cualquier discusión sobre multilingüismo debe tomar en cuenta el hecho de que toda interacción verbal está siempre regida por reglas, y que dichas reglas van más allá de las reglas gramaticales. La comunicación multilingüe requiere, por consiguiente, tanto una gramática social compartida, como reglas compartidas del uso de la variedad correspondiente. Dicha gramática, evocando a su homóloga generativa, llevaría implícita, según Gumperz (1969, pág. 244), la hipótesis de un conjunto general subyacente de reglas, que se aplicarían a todos los 
aspectos del comportamiento bilingüe. Las reglas no compartidas podrían considerarse como fenómenos de un orden inferior. Ahora bien, tal teoría sociolingüistica describiría la competencia del hablanteoyente real de una comunidad de habla heterogéneamente multilingüe y/o multilectal.

Asume, pues, esta dirección que la comunidad de habla bilingüe dispone de una compleja competencia comunicativa, que nos permite englobar las distintas variedades lingü ísticas, y así describir y explicar de un modo único el comportamiento de habla de tal comunidad. En tal sentido el repertorio lingüistico define el conjunto de los recursos lingüisticos disponibles por los miembros de una particular comunidad. El repertorio, en otras palabras, es el diasistema que nos describe la competencia comunicativa de los miembros bilingües de la comunidad, cada uno de los cuales, de acuerdo con el significado social y contextual, selecciona dentro del repertorio los sociolectos y registros pertinentes. Veamos un ejemplo, dados dos subsistemas ABC... de dos variedades $\left(L_{1}\right.$ y $\left.L_{2}\right)$ en alternancia, los cuales representamos como

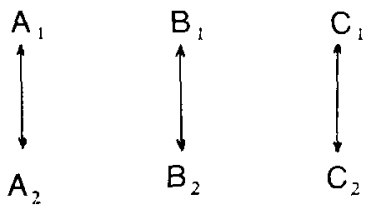

se pueden dar tres formulaciones del análisis bilingüe:

a) bilingüismo como coexistencia conflictiva de dos sistemas uniformes, y desde esta base se propone predecir su interacción (Fries y Pike, 1949);

b) bilingüismo como alternancia completa o nula de dos sistemas separados, cada uno unido por reglas de estricta coocurrencia, es decir, asistimos a un sistema de tipo único $A_{1} B_{1} C_{1} \circ A_{2} B_{2} C_{2}$, pero nunca $A_{1} B_{2} C_{1}, 0$

c) bilingüismo como alternancia entre dos subsistemas de un único repertorio, donde cualquier elemento puede covariar dentro de él, $p$. ej. la variable $(B)$ con variantes $B_{1}$ y $B_{2}$ puede covariar con otras variables $(A)$ y $(C)$, y podria darse, aunque raramente $A_{1} B_{2} C_{1}$, que justificaría hablar de alternancia o cambio de norma dentro de un único sistema complejo. Por otra parte, las interrelaciones sistemáticas de $A_{1}$, $B_{1}$ y $C_{1}$ se producen más por sus respuestas a las mismas variables independientes y por sus posibilidades de descripción en términos de 
las mismas funciones cuantitativas que por la dirección uniforme de la sustitución de norma bajo las mismas condiciones.

Pues bien, solamente en este último caso se puede describir de un modo único el comportamiento de habla de la comunidad bilingüe, en términos de su selección dentro de grupos particulares de variables lingüísticas o conjuntos de subsistemas gramaticales, $y$, de esta manera, se puede establecer el carácter sistemático de los dos polos $A_{1} B_{1}$ $C_{1} \ldots$ y $A_{2} B_{2} C_{2} \ldots$, y abrir el camino a estudios más profundos de la influencia mutua en el multilingüismo. No de otra manera se ofrece validez empírica a nociones descriptivas tales como estándar, geolecto, sociolecto..., puesto que se suscriben dichos modelos más que selecciones entre rasgos individuales (Weinreich, Labov y Herzog, 1968, págs. 166 ss.; Labov, 1969a, págs. 252 s.; v. Stewart, 1962; Gimeno, 1979, págs. 130 ss.).

El diasistema heterogéneo es, entonces, un conjunto de (sub)sistemas que alternan de acuerdo con un complejo de reglas interrelacionadas o coocurentes, delimitando claramente ambos (sub)sistemas. Ahora bien, dentro de cada uno de estos (sub)sistemas podemos encontrar variables lingüisticas individuales que no coocurran estrictamente. Cada una de dichas variables será definida en función del entorno lingüístico y contexto social, y normalmente podrá hallarse covariación interior entre ellas. La covariación, en tal caso, puede oponerse a la estricta coocurrencia, o la coocurrencia puede ser concebida como caso limite de covariación. Sin embargo, la demostración de tal covariación sistemática requeria una metodología más estricta que la simple hipótesis de que ciertas formas ocurrieran o no.

4.2. Labov (1970, págs. 237 ss.) ha expresado la especificación de la influencia diversa de los entornos lingü ísticos y contextos extralingüisticos - ya la variación social, ya la funcional-, a partir de una atención al discurso y una percepción de las normas, a fin de determinar la frecuencia de algunas variantes lingüísticas, llamadas variables sociolingüisticas, como unidades minimas no discretas que representen la variabilidad pronosticable y ordenable dentro del sistema heterogéneo. Dichas variables continuas no implican variantes continuas, puesto que se codifican dentro de un limitado conjunto de elecciones o variantes discretas; asi la variable $(B)$ aparece reducida a la variante $B_{1}$ y a la variante $B_{2}$, y será, por consiguiente, la frecuencia de la elección por parte de los hablantes la que producirá de un modo categórico la variable continua.

No todos los elementos del repertorio lingüístico son representati- 
vos para la distribución sociolingüística, y por tanto, no todos merecen una representación mediante una estructura sociolingüistica. Son estructuras sociolingüisticas las variables sociolingüisticas que están correlacionadas en forma significativa a variables extralingüisticas del contexto social. Variable sociolingüística será, pues, aquel rasgo lingüistico que se da en correlación con ciertos grupos sociales y algunas variables no lingüisticas del contexto social: hablante, oyente, marco, etc. En tal sentido, dicha variable engloba la dependencia reciproca de los grupos sociales y las variables funcionales, y se configura como una regla abstracta, o regla variable, de una cierta teoría sociolingüistica (v. Labov, 1969b; Cedergren y Sankoff, 1974; Sankoff, 1975; López Morales, 1977; Rousseau y Sankoff, 1978; Sank off y Labov, 1979; Gimeno, 1979, págs. 147 ss.; López Morales, 1981; Gimeno, 1981, págs. 366 ss.; Cedergren, 1983; López Morales, 1983).

4.2.1. En un sentido explicativo, la competencia sociolingüística recoge aquellos aspectos sistemáticos de la actuación del bilingüe de una comunidad de habla, a través de las reglas variables, donde se combinan directamente las técnicas transformatorias con un análisis cuantitativo de la variación regular e inherente a dicha comunidad bilingüe. Tales reglas caracterizarán a los componentes de un subgrupo determinado para descubrirnos la gramática social o comunitaria, que describe, de este modo, la variación presente en la comunidad de habla y pronostica con precisión el comportamiento de cada bilingüe.

La competencia sociolingüística, en suma, estará integrada por ese conjunto ordenado de reglas variables, que representan la covariación sistemática de los datos lingüísticos y las circunstancias sociales, bajo la forma de probabilidades teóricas, a fin de indicar la contribución de los rasgos variables del entorno gramatical a la aplicabilidad de dichas reglas. La actuación sociolingüística considerará las frecuencias observadas como reflejo estadístico de la competencia. Con otras palabras, asignamos las probabilidades de las reglas a la competencia sociolingüística y las frecuencias observadas de las reglas a la actuación sociolingüística, contemplando, de este modo, la explicabilidad a partir del estudio de las reglas variables (Labov, 1969b, págs. 757 ss.; Cedergren y Sankoff, 1974, pág. 343).

La teoria variacionista del multilingüismo y multilectismo se plantea, pues, la elaboración de unas gramáticas sociales que describan la variación presente en cualquier comunidad de habla, y pronostiquen con precisión el comportamiento de cada individuo. El propósito de estas gramáticas es caracterizar la competencia sociolingüística de los com- 
ponentes de un subgrupo determinado, a través de un sistema de reglas variables que representen la covariación de los factores lingüisticos y sociales, bajo la forma de probabilidades teóricas.

4.3. Ahora bien, la convergencia gramatical como proceso histórico sumamente complejo suscita algunas cuestiones de especial interés. Una de ellas es el llamado "cambio de norma". Las situaciones de divergencia lingüística dentro de una misma comunidad multilingüe o monolingüe se han mantenido en algunos lugares durante largos períodos de tiempo. La comunicación, como tal, responde a identidades culturales ${ }^{3}$ y a los valores sociales implicados, y resulta canalizada por límites sociales y políticos de control social. Los cambios de norma y de registro son en gran medida fenómenos socioculturales y juegan un importante papel en el entramado de la interacción social. La capacidad de cada hablante para seleccionar el cambio de norma o de registro adecuados en la situación concreta representa un indicador válido de su competencia sociolingüistica multilectal (cfr. Gumperz, 1967; Blom y Gumperz, 1968; Gumperz, 1969b; Várvaro, 1972/1973; Gumperz y Bennett, 1980).

Un estudio significativo de la situación de diglosia estable del español con el inglés en la comunidad de habla puertorriqueña de Nueva York nos la ofrece la investigación empírica de Poplack (1978; 1979; 1982a; 1982b; Sankoff y Poplack, 1981; Pousada y Poplack, 1982). La norma de interacción de la comunidad puertorriqueña es el cambio de norma, el cual resulta regido por restricciones lingüisticas, sociales y funcionales. Las restricciones sintácticas de morfema libre y de equivalencia, y los tres tipos de cambio de norma («etiqueta", oracional e intraoracional) nos muestran el mecanismo específico del cambio de norma y la estrategia del hablante para evitar enunciados agramaticales, en función de los diferentes grados de habilidad bilingüe.

La gramática del cambio de norma responderia a una simple gramática superficial independiente del contexto, la cual se basaría en dos

3 Una interpretación funcional del bilingüismo requiere colocarlo dentro de la perspectiva de una experiencia bicultural. De este modo, el biculturalismo constituirá algo más que dos niveles culturales coetáneos en la vida social del individuo, y será más bien un sistema de formas alternativas en estado de selección y sincretismo constantes para el uso y manipulación de una sola realidad orgánica -la del individuo_, y de una doble realidad superorgánica - la de las dos culturas-. En tales términos, la homogeneización cultural en el individuo implica homologar funcionalmente en su personalidad los elementos de una cultura con los de otra, haciéndose menos intensiva en cada una de ellas. El ego de un individuo bicultural es internamente más monocultural que lo es su sociedad (Esteva Fabregat, 1978, págs. 19 ss.). 
gramáticas monolingües y nos mostraría cómo el hablante mantiene estrictamente las distinciones cualitativas y cuantitativas entre la gramática española y la inglesa. Por otra parte, una aproximación cuantitativa y cualitativa al cambio de norma nos permiten un análisis más completo de la mayoría de los datos y una gramática probabilística del cambio de norma. En suma, el análisis del cambio de norma tiene profundas implicaciones para la teoría gramatical, e indica el modo en que dos lenguas pueden reconciliar sus diferencias, aunque sin modificarlas, hasta conseguir una forma de comunicación tan funcional como el habla monolingüe (Poplack, 1982, pág. 15).

La aproximación sociopsicológica a la conducta intercomunitaria, en general, y a las lenguas y etnias, en particular, debe tener en cuenta los aspectos de la teoría de la identidad social, los conceptos de percepción de la vitalidad etnolingüistica y de los límites de grupo, así como la noción de pertenencia como miembro a múltiples grupos. Una identidad étnica negativa y una conciencia de la posibilidad de alternativas cognitivas pueden provocar una o varias estrategias de movilidad social, creatividad social o competición social, las cuales tienen una importante correlación con una convergencia hacia las características lingüísticas del grupo externo y el «suicidio de la lengua» (Giles, 1981, págs. 77 ss.).

En un intento de ofrecer un análisis teórico de las relaciones entre lengua y grupo étnico, Giles, Bourhis y Taylor (1977) propusieron la integración de una taxonomía de los factores de vitalidad etnolingüistica y las teorias de relaciones intercomunitarias de Tajfel y de acomodación de habla de Giles. Se ha dicho que la vitalidad de un grupo es mayor cuantos más factores socioestructurales le sean favorables, $y$, por supuesto, tiene más probabilidades de supervivencia y de aparecer como entidad colectiva diferenciada. La acomodación de habla plantea unos procesos de convergencia, no convergencia o divergencia, y sugiere que los grupos están continuamente modificando su variedad para reducir, mantener o acentuar las diferencias lingüisticas (y sociales), dependiendo de su percepción del contexto de interacción.

4.3.1. Otra de las cuestiones especiales es la relacionada con el concepto del contínuum criollo y con el ámbito de las lenguas criollas. La formación del continuum criollo responde a un proceso de transculturación o aculturación. Su naturaleza auténtica reside en las formas característicamente peculiares de los mesolectos, y constituyen una prueba de una gramática polilectal o multilectal (v. Decamp, 1971; Bickerton, 1973b; Labov, 1973; Bickerton, 1975). La noción de gramática 
multilectal fue sugerida por Bailey (1972), quien señaió la posibilidad de escribir una gramática que englobara todos los lectos de una lengua, puesto que la competencia del nativo adulto trasciende la variedad que él mismo utiliza. Posteriormente, Bickerton ha pretendido demostrar que las gramáticas multilectales pueden escribirse, y ello no significaria el incremento de complejidad que podriamos haber temido. No obstante, nos encontrariamos con una gran dificultad, ya que las gramáticas existentes son todas monolectales y sólo se tienen ambiguas noticias sobre lo que son las propiedades estructurales de una gramática multilectal, y sobre cuál es, entre las posibles formas, la que podria adaptarse.

En particular, Bickerton (1973a) propone un modelo de gramática multilectal basado en una escala de implicación de los sistemas de reglas individuales, la cual nos permitiria plantear hipótesis sobre jerarquia de prioridad entre los rasgos, y así postular un orden provisional entre los datos aparentemente conflictivos que puedan servir para la escritura eventual de reglas formales. De esta manera tales reglas serian capaces de generar todas las variedades de la lengua en cualquier comunidad de habla que no sea inequivocamente bilingüe o multilingüe, es decir, donde haya un continuum de habla con una ruptura no claramente definida (pág. 20). El contínuum criollo, el contínuum geolectal y el contínuum temporal serían de facto aspectos diferentes de los procesos de transición e inserción lingüistica y social del cambio lingüistico (v. Gimeno, 1983a, págs. 196 ss.).

Por su parte, el Heidelberger Forschungsprojekt «Pidgin-Deutsch" (1978) supone una explicación altamente simplificada del proceso global de la adquisición de una segunda lengua por los inmigrantes extranjeros, en términos de una aproximación escalonada, que pasa a través de variedades intermedias o de transición hacia una variedad ideal. Ahora bien, estas gramáticas de transición son descriptivamente casos particulares de "gramáticas variacionales probabilisticas" de un espacio multidimensional de variedades, que constituyen una gramática de referencia ( $v$. una revisión funcionalista en Weydt y SchliebenLange, 1981).

4.4. En el pasado, he propuesto la posibilidad metodológica de aplicar el concepto de comunidad de habla al contexto comunicativo de las comarcas (valencianohablantes y murcianohablantes) del sur del $P a i s$ Valencià, a fin de determinar aquellas comunidades menores que se caracterizan por la interacción primaria y común de sus miembros. En este caso, el repertorio lingüístico reúne las actuaciones comunica- 
tivas de esa comunidad de habla, independientemente del hecho de que se utilice la misma lengua $u$ otra distinta. Concretamente, el repertorio lingüistico de la comunidad de habla alicantina identifica la competencia sociolingüística compleja del contexto comunicativo de las comarcas valencianas del sur de la frontera histórica Biar-Busot (excepto Villena y Sax), e integra las variedades sociales y funcionales presentes en esas comarcas, es decir, el sociolecto valenciano y el castellano (estándar y/o murciano seseante) (v. Montoya, 1981; Ros, 1982; Gimeno, 1984b; 1984c; 1984d).

La comunidad de habla alicantina ha sido y es una encrucijada lingüística, social y cultural, desde los primeros tiempos de la conquista islámica. Dentro del contexto valenciano se singulariza por un carácter marcadamente fronterizo, donde las barreras (socio)lingüisticas, históricas, geográficas y económicas sesgan cualquier pauta interna de conducta. Sin embargo, este hecho diferencial de las comarcas meridionales del Pais Valencià constituye su personalidad colectiva. La gramática social sincrónica de nuestra comunidad es multilectal, puesto que se basa en una covariación entre los dos (sub)sistemas de un único repertorio. Descriptivamente, la situación del lecto valenciano más meridional y del murciano seseante responden a variedades intermedias o "gramáticas de transición" - dependiendo del contexto social--, como casos particulares de gramáticas variacionales, a saber: a) valenciano-alicantina, que posee una competencia activa del sociolecto valenciano, y b) murciano-alicantina, que tiene una competencia pasiva del sociolecto valenciano.

Cada una de estas gramáticas caracteriza la variedad situada en cierto punto del proceso de sustitución lingüística, y muestra la distancia - mayor o menor, respectivamente- del lecto valenciano al castellano estándar (v. fig. 2).

La sustitución lingüística catalano-castellana (v. Aracil, 1966; Ninyoles, 1972), un cambio de norma (v.g. el seseo valenciano, v. Montoya, 1982; Gimeno, 1983b) y una gramática de transición (v.g. el análisis semántico de los verbos portar, dur y posar/llevar // ficar/traure, v. Colomina, 1983) parecen estar implicados en nuestro contexto social, a falta de una más precisa ratificación empírica. El análisis de regla variable y las escalas de implicación han resultado instrumentos fructiferos para sistematizar el estudio de la variación lingüística y modelar formalmente la competencia sociolingüistica multilectal de nuestra comunidad.

4.5. Aunque el modelo variacionista todavía no dispone de la correspondiente sanción de las deseables investigaciones empiricas, las 
críticas se han centrado sobre la asunción de las reglas variables como gramática de la comunidad, las cuales habrian sido más adoptadas tácitamente que demostradas teóricamente (Kay, 1978). Las reglas variables no darian cuenta de la interacción entre las restricciones sociales y lingüísticas, y, por tanto, no proveerían un modelo adecuado de las comunidades de habla en las cuales la heterogeneidad lingüistica presente fuera parte de un cambio lingüistico en progreso ( $v$. Romaine, 1982, págs. 234 ss.).

Diasistema catalán

Diasistema castellano

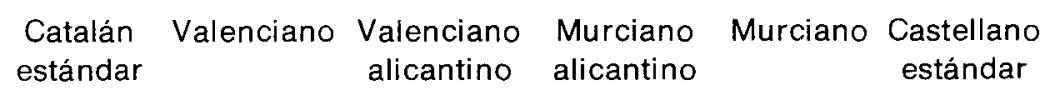

Fig.2.-Situación descriptiva de los lectos variacionales alicantinos dentro del continuum geolectal (y social) catalano-castellano.

\section{Reflexiones finales}

Hemos visto, pues, tres direcciones que persiguen una misma meta: el análisis de las comunidades multilingües y multilectales. Ciertamente son tres caminos, y como tales, uno puede preguntarse cuál decidir, cuál escoger. Es más, no creo que esté dicha la última palabra sobre multilingüismo y multilectismo y, quizá, no esté la solución en ninguno de los tres, o en los tres conjuntamente. Es decir, dada la propia progresión de la investigación multilingüe (y multilectal) y la peculiar contribución de cada una de las etapas, la superación orientativa y la complementariedad o suplementariedad entre ellas podría ser una nueva vía, sin hipotecar por supuesto la propia línea teórica y metodológica.

Los estudios multilingües y multilectales, debido a sus peculiares caracteristicas, implican un tratamiento sociolingüistico general o in- 
terdisciplinario, $y$, además, son dependientes de los modelos pragmáticos de actuación comunicativa, como de una psicolingüística. Por otro lado, los conceptos de regla variable y escala de implicación parecen ser los más prometedores modelos explicativos de la comunidad de habla multilingüe y/o multilectal. Quiero decir, en resumen, que una sociolingüística del multilingüismo y multilectismo deberá de hacer factible una metodología estricta y otra general con las aportaciones pragmáticas y psicolingüisticas. La auténtica dimensión del asunto residirá, lejos del aislacionismo y dogmatismo, en partir de unos supuestos de diferenciación e integración, y en la constitución de una teoría sociolingüistica que explique la competencia sociolingüistica de la comunidad de habla multilingüe y/o multilectal.

\section{Referencias bibliográficas}

Alpera, LI. (1981), "Cap a una interpretació sociolingüística i semàntica dels problemes d'interferències i de substitucions lèxiques en el valencià meridional», Item, 5 págs. 93-106.

Aracil, LI. V. (1965), Conflit linguistique et normalisation linguistique dans l'Europe nouvelle, reed., Perpignan: I.R.S.C.E., 1976.

- (1966), "Un dilema valencià», Identity, Cambridge, Mass., 24, págs. 17-29.

- (1979a), "Substitució lingüistica», en Papers de sociolingüística, Barcelona, 1982, págs. 115-20.

- (1979b), "Educació i sociolingüistica», Treballs de Sociolingüistica Catalana, 2, págs. 33-86.

- (1982), Papers de sociolingüistica, Barcelona: La Magrana.

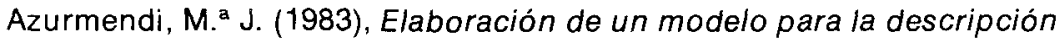
sociolingüistica del bilingüismo y su aplicación parcial en la comarca de San Sebastián, 3 vols., San Sebastián: Caja de Ahorros Provincial de Guipúzcoa.

Badia i Margarit, A. M. (1964), Llengua i cultura als Països Catalans, 3. ed., Barcelona: Edicions 62, 1972.

- (1969), La llengua dels barcelonins: Resultats d'una enquesta sociològico-lingüística, I, Barcelona: Edicions 62.

- (1972), «Langue et société dans le domaine linguistique catalan, notamment à Barcelone», Revue de Linguistique Romane, 36, 143-144, págs. 263-304.

- (1974), «Entorn de la sociolingüística catalana: Precedents, dificultats, contingut, objectius», Treballs de Sociolingüistica Catalana, 1, 1977, págs. 15-35.

- (1977), "Lenguas en contacto: Bilingüismo, diglosia, lenguas en 
convivencia (Con especial aplicación al catalán)», en Lapesa R. (coord.), Comunicación y lenguaje, Madrid: Karpos, págs. 109-33.

Bailey, Ch.-J. N. (1972), "The Integration of Linguistic Theory: Internal Reconstructions and the Comparative Method in Descriptive Analysis», en Stockwell, R. P. y R. K. S. Macaulay (eds.), Linguistic Change and Generative Theory, Bloomington-London: Indiana University Press, págs. 22-31.

- (1973), Variation and Linguistic Theory, Arlington, Virginia: Center for Applied Linguistics.

Bickerton, D. (1973a), "The Structure of Polyectal Grammars", en Shuy, R. W. (ed.), Sociolinguistics: Current Trends and Prospects, Washington, D.C., págs. 17-42.

- (1973b), "The Nature of a Creole Continuum», Language, 49, 3, págs. 640-69.

- (1975), Dynamics of a Creole System, Cambridge: Cambridge University Press.

Blom, J.-P. y J. J. Gumperz (1968), «Social Meaning in Linguistic Structures: Code-Switching in Norway", en Gumperz, J. J. y D. Hymes (eds.), Directions in Sociolinguistics. The Ethnography of Communication, New York: Holt, Rinehart and Winston, 1972, págs. 407-34.

Bloomfield, L. (1933), Language, New York: H. Holt. Trad. esp. El lenguaje, Lima: Universidad Nacional Mayor de San Marcos, 1964.

Cedergren, H. J. (1983), "Sociolingüística", en López Morales, H. (coord.), Introducción a la lingüistica actual, Madrid, págs. 147-65. Cedergren, H. y D. Sankoff (1974), "Variable Rules: Performance as a Statistical Reflection of Competence», Language, 50, págs. 333-55. Colomina, J. (1983), De la dialectologia a la teoria de la variació lingüistica: El cas del valencià meridional alacanti, Tesis doctoral, Facultad de Filosofía y Letras, Universidad de Alicante (inédita).

Coseriu, E. (1981), «La socio- y la etnolingü istica: sus fundamentos y sus tareas", Anuario de Letras, 19, págs. 5-30.

Decamp, D. (1971), "Implicational Scales and Sociolinguistic Linearity", Linguistics, 73 , págs. 30-43.

Diebold, A. R. Jr. (1961), “Incipient Bilingualism», Language, 37, págs. 97-112.

Dittmar, N. (1973), Sociolinguistics. A Critical Survey of Theory and Application, Londres: E. Arnold, 1976.

Esteva Fabregat, C. (1978), «El biculturalismo como contexto del bilingüismo", en I.C.E. de la Universidad de Barcelona (ed.), Bilingüismo y biculturalismo, Barcelona: Ceac, págs. 9-51.

Ferguson, Ch. A. (1959), «Diglossia», Word, 15, págs. 325-40. 
Fishman, J. A. (1968a), «Sociolinguistic Perspective on the Study of Bilingualism», en Fishman, J. A., R. L. Cooper, R. Ma et al., Bilingualism in the Barrio, Bloomington, 1975, págs. 557-82.

- (1968b), "Societal Bilingualism: Stable and Transitional», en Dil, A. S. (ed.), Language in Sociocultural Change. Essays by J. A. Fishman, Stanford, California: Stanford University Press, 1972, págs. 135-52.

- (1969), «The Description of Societal Bilingualism», en Kelly, L. G. (ed.), Description and Measurement of Bilingualism, Toronto, págs. 275-81.

Fishman, J. A., R. L. Cooper, R. Ma et al. (1971), Bilingualism in the Barrio, 2. ${ }^{a}$ edn. rev., Bloomington: Indiana University Publications, 1975.

Fries, Ch. C. y K. L. Pike (1949), “Coexistent Phonemic Systems», Language, 25, págs. 29-50.

Fuster, J. (1962), Nosaltres els valencians, 2. ${ }^{a}$ edn., Barcelona: Edicions 62, 1964.

Giles, H. y J. L. Byrne (1981), "Aproximación intercomunitaria a la adquisición de la segunda lengua», Revista de Educación (Madrid), 268, págs. 77-102.

Giles, H., R. Y. Bourhis y D. M. Taylor (1977), "Towards a Theory of Language in Ethnic Group Relations», en Giles, H. (ed.), Language, Ethnicity and Intergroup Relations, New York: Academic Press, págs. 307-48.

Gimeno, F. (1975), Sociolingüística catalana: El habla marinera de Santa Pola, Tesis doctoral, Facultad de Filosofía y Letras, Universidad de Murcia (inédita).

- (1977), «Introducción a la sociolingüistica», Item (Alicante), 2, págs. 53-65.

- (1979), «Sociolingüística: Un modelo teórico», Boletín de la Academia Puertorriqueña de la Lengua Española, 7, 1, págs. 125-68.

- (1981), "Dimensiones del multilingüismo", Revista Española de Lingüistica, 11, 2, págs. 341-73.

- (1982a), "Alternatives al bilingüisme valencià meridional: A proposit d'una pre-enquesta sobre les actituds lingüistiques", Treballs de Sociolingüistica Catalana, 4, págs. 51-61.

- (1982b), «Aproximación sociolingüística a la comunidad de habla valenciana meridional», en Ninyoles, R. LI. (ed.), Estructura social al Pais Valencià, València: Diputació, págs. 725-37.

- (1983a), "Hacia una sociolingüistica histórica», Estudios de Lingüística de la Universidad de Alicante, 1, págs. 181-226.

— (1983b), "El seseo valenciano de la comunidad de habla alican- 
tina», Anales de Literatura Española de la Universidad de Alicante, 1 (1982), págs. 345-62.

Gimeno, F. (1983c), "Vers una sociolingüística historica?", Treballs de Sociolingüistica Catalana, 5, págs. 53-70.

- (1984a), “Estudio sociolingüistico histórico», en Estal, J. M. del, M. ${ }^{a}$ L. Cabanes y F. Gimeno (eds.), Libro de los primitivos privilegios de Alicante de Alfonso $X$ el Sabio, edn. facsimilar con introd. de -, Madrid: Edilán, págs. 13-6.

- (1984b), «El sutrato lingüístico y el seseo valenciano de la comunidad de habla alicantina", Cuadernos de Filologia (Homenaje a $M$. Sanchis Guarner) (en prensa).

- (1984c), "Die Mundart von Alacant i el bilingüisme alacantí de l'àrea Alacantí-Vinalopó", Actes del I Congrés d'Estudis del Camp d'Alacant, Alicante, 29 de marzo-2 de abril de 1982 (en prensa).

- 1984d), «A propósito de comunidad de habla: "The Social Dimension of Dialectology", de J. P. Rona", Actas del I Congreso Internacional sobre el Español de América, San Juan, 4-9 de octubre de 1982 (en prensa).

- (1985), "En torno a dos traslados romances del estilo cancilleresco de Alfonso X", Actas del Congreso Internacional con motivo del VII centenario de la muerte de Alfonso X (1284-1984), Madrid-Sevilla, 29 de marzo-4 de abril de 1984 (en prensa).

Granda, G. de (1978), Estudios lingüísticos hispánicos, afrohispánicos y criollos, Madrid: Gredos.

- (1980), «Préstamos morfológicos del guaraní en el español de Paraguay" I (Revue de Lingüistique Romane, 44, 173-174, págs. 57-68), II (Revue de Linguistique Romane, 44, 175-176, págs. 247-65).

Gumperz, J. J. (1967), «On the Linguistic Markers of Bilingual Communication», Journal of Social Issues, 23, 2, págs. 48-57.

- (1969a), «How Can We Describe and Measure the Behavior of Bilingual Groups?», en Kelly, L. G. (ed.), Description and Measurement of Bilingualism, Toronto, págs. 241-58.

- (1969b), "Communication in Multilingual Societies", en Tyler, S. (ed.), Cognitive Anthropology, New York. Reed. en Dil, A. S. (ed.), Language in Social Groups. Essays by J. J. Gumperz, Stanford, Calif.: Stanford University Press, 1971, págs. 230-47.

- (1970), "Sociolinguistics and Communication in Small Groups", en Pride, J. B. y J. Holmes (eds.), Sociolinguistics, Harmondsworth: Penguin Books, 1972, págs. 203-24.

Gumperz, J. J. y A. Bennett (1980), "Language and Culture», en People 
in Culture. A Survey of Cultural Anthropology, New York. Trad. esp. Lenguaje y cultura, Barcelona: Anagrama, 1981.

Gumperz, J. J. y E. Hernández (1971), «Bilingualism, Bidialectalism and Classroom Interaction", en Dil, A. S. (ed.), Language in Social Groups. Essays by J. J. Gumperz, Stanford, Calif.: Stanford University Press, págs. 311-39.

Haugen, E. (1953), The Norwegian Language in America. A Study in Bilingual Behavior, 2. ${ }^{a}$ edn., Bloomington: Indiana University Press, 1969.

- (1973), «Bilingualism, Language Contact and Immigrant Languages in the United States: A Research Report 1956-70", en Sebeok, T. A. (ed.), Current Trends in Linguistics. Linguistics in North America, 10, I, The Hague-Paris: Mouton, págs. 505-91.

Heidelberger Forschungsprojekt «Pidgin-Deutsch» (1978), «The Acquisition of German Syntax by Foreign Migrant Workers», en Sankoff, D. (ed.), Linguistic Variation, New York, págs. 1-22.

Kay, P. (1978), "Variable Rules, Community Grammar, and Linguistic Change", en Sankoff, D. (ed.), Linguistic Variation, New York, págs. 71-83.

Kelly, L. G. (ed.) (1969), Description and Measurement of Bilingualism: An International Seminar, Universidad de Moncton, 6-14 de junio de 1967, Toronto: Toronto University Press.

Kloss, H. (1967), "Bilingualism and Nationalism", The Journal of Social Issues, 23, 2, págs. 39-47.

Kremnitz, G. (1979), "La sociolingüistica catalana», Treballs de Sociolingüistica Catalana, 3, págs. 135-57.

Labov, W. (1969a), "Commentaries", en Kelly, L. G. (ed.), Description and Measurement of Bilingualism, Toronto, págs. 250-5.

- (1969b), «Contraction, Deletion and Inherent Variability of the English Copula", Language, 45, 4, págs. 715-62.

- (1970), "The Study of Language in Its Social Context", en Sociolinguistic Patterns, Oxford: B. Blackwell, 1978, págs. 183-259.

- (1973), "Were do Grammars Stop?", en Shuy, R. W. (ed.), Sociolinguistics: Current Trends and Prospects, Washington, D.C., págs. 43-88.

Lafont, R. (1977), «Sobre el procés de patoisització", Treballs de Sociolingüistica Catalana, 1, págs. 131-6.

López Morales, H. (1977), "Sociolingüistica: Nuevos enfoques metodológicos", Revista Española de Lingüistica, 7, 2, págs. 17-36.

- (1981), «Estudio de la competencia sociolingü istica: los modelos probabilísticos", Revista Española de Lingüistica, 11, 2, págs. 247-68. 
López Morales, H. (1983), «Lingüistica estadistica», en López Morales, H. (coord.), Introducción a la lingü istica actual, Madrid, págs. 209-25. Mackey, W. F. (1962), "The Description of Bilingualism», en Fishman, J. A. (ed.), Readings in the Sociology of Language, The HagueParis: Mouton, 1972, págs. 554-84.

- (1969), "Conclusión", en Kelly, L. G. (ed.), Description and Measurement of Biligualism, Toronto, págs. 361-6.

- (1976), Bilinguisme et contact des langues, Paris: editions Klincksieck.

Montoya, B. (1981), Confluència de llengües a les Valls del Vinalopó (Un cas de sociolingüistica valenciana), Memoria de Licenciatura, Facultad de Filosofía y Letras, Universidad de Alicante (inédita).

- (1982), "Los islotes lingüisticos de Aspe y Elda en el Vinalopó Medio", Revista del Instituto de Estudios Alicantinos, 35, págs. 119-34.

Ninyoles, R. LI. (1972), Idioma y poder social, Madrid: Editorial Tecnos.

- (1976a), "Idéologies diglossiques et assimilation", en Ninyoles, R. LI. (ed.), Estructura social al País Valencià, València: Diputació, 1982, págs. 669-77.

- (1976b), Bases per a una política lingüistica democràtica a l'Estat Espanyol, València: Tres i Quatre.

- (1977), Cuatro idiomas para un estado (El castellano y los conflictos lingüisticos en la España periférica), Madrid: Cambio 16.

- (1981), «La política lingüistica: modelos y ámbitos», en Ninyoles, R. Ll. et al., Las lenguas nacionales en el ámbito de la Administración, Valencia: Diputación Provincial, págs. 15-39.

Oksaar, E. (1972), «Bilingualism», en Sebeok, T. A. (ed.), Current Trends in Linguistics. Linguistics in Western Europe, 9, I, The HagueParis: Mouton, págs. 476-511.

Poplack, S. (1978), "Syntactic Structure and Social Function of CodeSwitching", en Durán, R. (ed.), Latino Language and Communication, Norwood, New Jersey: Ablex, 1981, págs. 169-84.

- (1979), "Sometimes I'll Start a Sentence in Spanish Y TERMINO EN ESPAÑOL: Toward a Typology of Code-Switching", Linguistics, 18, 1980, págs. 581-618.

- (1982a), "Bilingualism and the Vernacular», en Hartford, B. et al. (eds.), Issues in International Bilingual Education. The Role of the Vernacular, New York: Plenum Press, págs. 1-23.

- (1982b), «Bilingual Competence: Linguistic Interference or Grammatical Integrity?», en Elias-Olivares, L. (ed.), Spanish in the U.S. Setting, National Clearinghouse for Bilingual Education (en prensa). 
Pousada, A. y S. Poplack (1982), “No Case for Convergence: The Puerto Rican Spanish Verb System in a Language-Contact Situation", en Fishman, J. A. y G. D. Keller (eds.), Bilingual Education for Hispanic Students in the United States, New York: Teachers College Press, págs. 207-37.

Quilis, A. et al. (1982), Interferencias lingüisticas en el habla de los niños españoles emigrantes en Francia, Madrid: Ministerio de Educación y Ciencia.

Romaine, S. (1982), Socio-historical Linguistics. Its Status and Methodology, Cambridge: Cambridge University Pres\$.

Ros, M. (1982), «Percepción y evaluación social de hablantes de cinco variedades lingüísticas", en Ninyoles, R. LI. (ed.), Estructura social al País Valencià, València: Diputació, págs. 679-97.

Rousseau, P. y D. Sankoff (1978), «Advances in Variable Rule Methodology", en Sankoff, D. (ed.), Linguistic Variation. Models and Methods, New York: Academic Press, págs. 57-69.

Sankoff, D. (1975), VARBRUL 2, Programa y documentación inéditos.

Sankoff, D. y W. Labov (1979), "On the Uses of Variable Rules", Language in Society, 8, págs. 189-222.

Sankoff, D. y S. Poplack (1981), «A Formal Grammar for CodeSwitching», Papers in Linguistics, 14, 2, págs. 3-46.

Schuchardt, H. (1885), Über die Lautgesetze. Gegen die Junggrammatiker, Berlin.

Shuy, R. S. (ed.) (1973), Sociolinguistics: Current Trends and Prospects. Report of the 23rd Annual Round Table Meeting on Linguistics and Language Studies, Washington, D.C.: Georgetown University Press.

Stewart, W. A. (1962), «A Sociolinguistic Typology for Describing National Multilingualism", vers. rev., en Fishman, J. A. (ed.), Readings in the Sociology of Language, The Hague-Paris: Mouton, 1972, págs. 531-45.

Vallverdú, F. (1970), Dues llengües: dues funcions? (La història contemporània de Catalunya des. d'un punt de vista sociolingüistic), 2. ${ }^{a}$ edn. rev. y act., Barcelona: Edicions 62, 1979.

- (1973), El fet lingüistic com a fet social (Assaig de lingüistica institucional), Barcelona: Edicions 62.

- (1980), Aproximació critica a la sociolingüistica catalana. Balanç dels estudis de sociologia lingüistica als Països Catalans, BarceIona: Edicions 62.

- (1981), El conflicto lingüístico en Cataluña: historia y presente, Barcelona: Península.

Várvaro, A. (1972/1973), «Storia della lingua: passato e prospettive di 
una categoria controversa", I (Romance Philology, 26, 1, 1972, págs. 16-51), II (Romance Philology, 26, 3, 1973, págs. 509-31). Vildomec, V. (1963), Multilingualism. General Linguistics and Psychology of Speech, Leyden: A. W. Sythoff.

Weinreich, U. (1953), Languages in Contact. Findings and Problems, 6 . $^{a}$ edn., The Hague-Paris: Mouton, 1968. Trad. esp. Lenguas en contacto. Descubrimientos y problemas, Venezuela: Ediciones de la Biblioteca de la Universidad Central, 1974.

- (1958), "On the Compatibility of Genetic Relationship and Convergent Development», Word, 14, págs. 374-9.

- (1968), «Unilinguisme et multilinguisme», en Martinet, A. (ed.), Le langage, Encyclopédie de la Pléiade, Paris: Éditions Gallimard, págs. $647-83$.

Weinreich, U., W. Labov y M. I. Herzog (1968), «Empirical Foundations for a Theory of Language Change», en Lehmann, W. P. y Y. Malkiel (eds.), Directions for Historical Linguistics: A Symposium, Austin: University of Texas Press, págs. 95-195.

Weydt, H. y B. Schlieben-Lange (1981), "Wie realistisch sind Variationsgrammatiken?», en Geckeler, H., B. Schlieben-Lange, J. Trabant y H. Weydt (eds.), Logos Semantikos, V, Madrid y Berlin: Gredos y W. de Gruyter, págs. 117-45. 